\title{
Existence result for semilinear elliptic systems involving critical exponents
}

\author{
S Khademloo*, M Farzinejad and O Khazaee kohpar
}

${ }^{\text {*Correspondence: }}$

s.khademloo@nit.ac.ir

Department of Basic Sciences,

Babol University of Technology,

Babol, 47148-71167, Iran

\begin{abstract}
In this paper we deal with the existence of a positive solution for a class of semilinear systems of multi-singular elliptic equations which involve Sobolev critical exponents. In fact, by the analytic techniques and variational methods, we prove that there exists at least one positive solution for the system.
\end{abstract}

MSC: $35 \mathrm{~J} 60 ; 35 \mathrm{~B} 33$

Keywords: semilinear elliptic system; nontrivial solution; critical exponent; variational method

\section{Introduction}

We consider the following elliptic system:

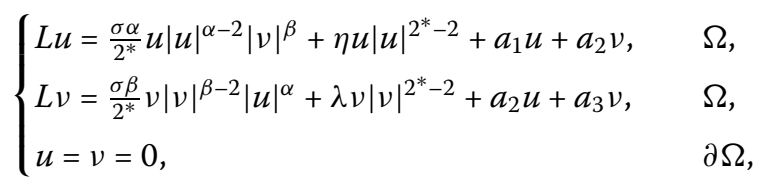

where $\Omega \subset \mathbb{R}^{N}(N \geq 3)$ is a smooth bounded domain such that $\xi_{i} \in \Omega, i=1,2, \ldots, k, k \geq 2$, are different points, $0 \leq \mu_{i}<\bar{\mu}:=\left(\frac{N-2}{2}\right)^{2}, L:=-\Delta \cdot-\sum_{i=1}^{k} \mu_{i} \frac{\cdot}{\left|x-\xi_{i}\right|^{2}}, \eta, \lambda, \sigma \geq 0, a_{1}, a_{2}, a_{3} \in$ $\mathbb{R}, 1<\alpha, \beta<2^{*}-1, \alpha+\beta=2^{*}$.

We work in the product space $H \times H$, where the space $H:=H_{0}^{1}(\Omega)$ is the completion of $C_{0}^{\infty}(\Omega)$ with respect to the norm $\left(\int_{\Omega}|\nabla \cdot|^{2} d x\right)^{\frac{1}{2}}$.

In resent years many publications [1-3] concerning semilinear elliptic equations involving singular points and the critical Sobolev exponent have appeared. Particularly in the last decade or so, many authors used the variational method and analytic techniques to study the existence of positive solutions of systems of the form of (1.1) or its variations; see, for example, [4-8].

Before stating the main result, we clarify some terminology. Since our method is variational in nature, we need to define the energy functional of (1.1) on $H \times H$

$$
\begin{aligned}
J(u, v)= & \frac{1}{2} \int_{\Omega}\left(|\nabla u|^{2}+|\nabla v|^{2}-\sum_{i=1}^{k} \frac{\mu_{i}\left(u^{2}+v^{2}\right)}{\left|x-\xi_{i}\right|^{2}}\right) d x \\
& -\frac{1}{2} \int_{\Omega}\left(a_{1} u^{2}+2 a_{2} u v+a_{3} v^{2}\right) d x-\sigma \frac{\sigma}{2^{*}} \int_{\Omega}|u|^{\alpha}|u|^{\beta} d x \\
& -\frac{\eta}{2^{*}} \int_{\Omega}|u|^{2^{*}} d x-\frac{\lambda}{2^{*}} \int_{\Omega}|v|^{2^{*}} d x .
\end{aligned}
$$

(0) 2012 Khademloo et al:; licensee Springer. This is an Open Access article distributed under the terms of the Creative Commons Attribution License (http://creativecommons.org/licenses/by/2.0), which permits unrestricted use, distribution, and reproduction in any medium, provided the original work is properly cited. 
Then $J(u, v)$ belongs to $C^{1}(H \times H, \mathbb{R})$. A pair of functions $\left(u_{0}, v_{0}\right) \in H \times H$ is said to be a solution of $(1.1)$ if $\left(u_{0}, v_{0}\right) \neq(0,0)$, and for all $(\varphi, \phi) \in H \times H$, we have

$$
\begin{aligned}
& \int_{\Omega}\left(\nabla u_{0} \cdot \nabla \varphi+\nabla v_{0} \cdot \nabla \phi-\sum_{i=1}^{k} \frac{\mu_{i}\left(u_{0} \varphi+v_{0} \phi\right)}{\left|x-\xi_{i}\right|^{2}}-\left(a_{1} u_{0} \varphi+a_{2} \varphi v_{0}+a_{2} \phi u_{0}+a_{3} v_{0} \phi\right)\right. \\
& \quad-\sigma\left(\alpha \varphi u_{0}\left|u_{0}\right|^{\alpha-2}\left|v_{0}\right|^{\beta}+\beta \phi v_{0}\left|v_{0}\right|^{\beta-2}\left|u_{0}\right|^{\alpha}\right) \\
& \left.\quad-\eta\left|u_{0}\right|^{2^{*}-2} u_{0} \varphi-\lambda\left|v_{0}\right|^{2^{*}-2} v_{0} \phi\right) d x=\left\langle J^{\prime}\left(u_{0}, v_{0}\right),(\varphi, \phi)\right\rangle=0 .
\end{aligned}
$$

Standard elliptic arguments show that

$$
u, v \in C^{2}\left(\Omega \backslash\left\{\xi_{1}, \ldots, \xi_{k}\right\}\right) \cap C^{1}\left(\bar{\Omega} \backslash\left\{\xi_{1}, \ldots, \xi_{k}\right\}\right)
$$

The following assumptions are needed:

$\left(\mathcal{H}_{1}\right) \eta+\lambda+\sigma>0,0 \leq \mu_{1} \leq \mu_{2} \leq \cdots \leq \mu_{k}<\bar{\mu}-1$ and $\sum_{i=1}^{k} \mu_{i}<\bar{\mu}, \alpha+\beta>1, \alpha+\beta=2^{*}$, $a_{1}, a_{2}, a_{3}>0, a_{1} a_{3}-a_{2}^{2}>0$

$\left(\mathcal{H}_{2}\right) \quad 0<\lambda_{1} \leq \lambda_{2}<\Lambda_{1}(\mu)$, where $\Lambda_{1}(\mu)$ is the first eigenvalue of $L, \lambda_{1}, \lambda_{2}$ are the eigenvalues of the matrix $A=\left(\begin{array}{ll}a_{1} & a_{2} \\ a_{2} & a_{3}\end{array}\right)$.

The quadratic from $Q(u, v):=(u, v) A(u, v)^{T}=a_{1} u^{2}+2 a_{2} u v+a_{3} v^{2}$ is positively defined and satisfies

$$
\lambda_{1}\left(u^{2}+v^{2}\right) \leq a_{1} u^{2}+2 a_{2} u v+a_{3} v^{2} \leq \lambda_{2}\left(u^{2}+v^{2}\right) \quad \forall u, v \in H
$$

Our main results are as follows.

Theorem 1.1 Suppose $\left(\mathcal{H}_{1}\right)$ holds. Then for any solution $(u, v) \in H \times H$ of problem (1.1), there exists a positive constant $C_{1}$ such that

$$
\max \{|u(x)|,|v(x)|\} \leq C_{1}\left|x-\xi_{i}\right|^{-\left(\sqrt{\bar{\mu}}-\sqrt{\bar{\mu}-\mu_{i}}\right)}, \quad \forall x \in B_{\rho_{1}}\left(\xi_{i}\right) \backslash\left\{\xi_{i}\right\}
$$

where $\rho_{1}>0$ and $B_{\rho_{1}}\left(\xi_{i}\right) \subset \Omega$.

Theorem 1.2 Suppose $\left(\mathcal{H}_{1}\right)$ holds. Then for any positive solution $(u, v) \in H \times H$ of problem (1.1), there exists a positive constant $C_{2}$ such that $B_{\rho_{2}}\left(\xi_{i}\right) \subset \Omega$ and

$$
\min \{u(x), v(x)\} \geq C_{2}\left|x-\xi_{i}\right|^{-\left(\sqrt{\bar{\mu}}-\sqrt{\bar{\mu}-\mu_{i}}\right)}, \quad \forall x \in B_{\rho_{2}}\left(\xi_{i}\right) \backslash\left\{\xi_{i}\right\}
$$

where $\rho_{2}>0$.

Theorem 1.3 Suppose $\left(\mathcal{H}_{1}\right),\left(\mathcal{H}_{2}\right)$ hold. Then the problem $(1.1)$ has a positive solution.

\section{Preliminaries}

On $H \times H$, we use the norm

$$
\|(u, v)\|_{H \times H}=\left(\int_{\Omega}\left(|\nabla u|^{2}+|\nabla v|^{2}\right) d x\right)^{\frac{1}{2}} .
$$


Using the Young inequality, the following best constant is well defined:

$$
S_{\mu_{i}}:=\inf _{u \in D^{1,2}\left(\mathbb{R}^{N}\right) \backslash\{0\}} \frac{\int_{\mathbb{R}^{N}}\left(|\nabla u|^{2}-\mu_{i} \frac{u^{2}}{\left|x-\xi_{i}\right|^{2}}\right) d x}{\left(\int_{\mathbb{R}^{N}}|u|^{2^{*}} d x\right)^{2 / 2^{*}}}
$$

where $D^{1,2}\left(\mathbb{R}^{N}\right)$ is the completion of $C_{0}^{\infty}\left(\mathbb{R}^{N}\right)$ with respect to the norm $\left(\int_{\mathbb{R}^{N}}|\nabla \cdot|^{2} d x\right)^{\frac{1}{2}}$. We infer that $S_{\mu_{i}}$ is attained in $\mathbb{R}^{N}$ by the functions

$$
V_{\mu_{i}, \varepsilon}^{\xi_{i}}(x)=\varepsilon^{\frac{2-N}{2}} U_{\mu_{i}}\left(\frac{x-\xi_{i}}{\varepsilon}\right), \quad \forall \varepsilon>0
$$

where

$$
U_{\mu_{i}}\left(x-\xi_{i}\right)=\frac{\left(\frac{2 N\left(\bar{\mu}-\mu_{i}\right)}{\sqrt{\bar{\mu}}}\right)^{\sqrt{\mu} / 2}}{\left|x-\xi_{i}\right|^{\left(\sqrt{\bar{\mu}}-\sqrt{\bar{\mu}-\mu_{i}}\right)}\left(1+\left|x-\xi_{i}\right|^{\frac{2 \sqrt{\bar{\mu}-\mu_{i}}}{\sqrt{\bar{\mu}}}}\right)^{\sqrt{\mu}}} .
$$

For all $\eta, \lambda, \sigma \geq 0, \eta+\lambda+\sigma>0, \alpha+\beta>1, \alpha+\beta=2^{*}$, by the Young and Hardy-Sobolev inequalities, the following constant is well defined on $\mathcal{D}:=\left(D^{1,2}\left(\mathbb{R}^{N}\right) \backslash\{0\}\right)^{2}$ :

$$
S_{\eta, \lambda, \sigma}\left(\mu_{i}\right):=\inf _{(u, v) \in \mathcal{D}} \frac{\int_{\mathbb{R}^{N}}\left(|\nabla u|^{2}+|\nabla v|^{2}-\mu_{i} \frac{u^{2}+v^{2}}{\left|x-\xi_{i}\right|^{2}}\right) d x}{\left(\int_{\mathbb{R}^{N}}\left(\eta|u|^{2^{*}}+\lambda|v|^{2^{*}}+\sigma|u|^{\alpha}|\nu|^{\beta}\right) d x\right)^{2 / 2^{*}}} .
$$

Set

$$
u_{\mu, \varepsilon}^{\xi}(x)=\psi(x) V_{\mu, \varepsilon}^{\xi}(x)=\varepsilon^{\frac{2-N}{2}} \psi(x) U_{\mu}\left(\frac{x-\xi}{\varepsilon}\right)
$$

where $\xi \in \Omega, 0 \leq \mu<\bar{\mu}, \psi \in C_{0}^{\infty}\left(B_{\rho}(\xi)\right)$ satisfies $0 \leq \psi \leq 1$ and $\psi \equiv 1, \forall x \in B_{\frac{\rho}{2}(\xi)}$, for all $\rho>0$ small. Then for any $0<\mu<\bar{\mu}$, by [9] we have the following estimates:

$$
\begin{aligned}
& \int_{\Omega}\left(\left|\nabla u_{\mu, \varepsilon}^{\xi}\right|^{2}-\mu \frac{\left(u_{\mu, \varepsilon}^{\xi}\right)^{2}}{|x-\xi|^{2}}\right) d x=S_{\mu}^{\frac{N}{2}}+o\left(\varepsilon^{2 \sqrt{\bar{\mu}}-\mu}\right), \\
& \int_{\Omega}\left|u_{\mu, \varepsilon}^{\xi}\right|^{2^{*}} d x=S_{\mu}^{\frac{N}{2}}+o\left(\varepsilon^{\frac{2 N}{N-2} \sqrt{\mu}-\mu}\right),
\end{aligned}
$$

and for any $a \in \mathbb{R}^{N} \backslash\{0\}$,

$$
\begin{gathered}
\int_{\Omega} \frac{\left|u_{\mu, \varepsilon}^{0}\right|^{2}}{|x+\xi|^{2}} d x= \begin{cases}\frac{\varepsilon^{2}}{|\xi|^{2}} \int_{\mathbb{R}^{N}} U_{\mu}^{2}(x) d x+o\left(\varepsilon^{2}\right) & \text { if } \mu<\bar{\mu}-1, \\
\frac{C_{\mu}^{2} \omega_{N}}{|\xi|^{2}} \varepsilon^{2}|\log \varepsilon|+o\left(\varepsilon^{2}\right) & \text { if } \mu=\bar{\mu}-1,\end{cases} \\
\int_{\Omega}\left|u_{\mu, \varepsilon}^{\xi}\right|^{2} d x= \begin{cases}o_{1}\left(\varepsilon^{2}\right) & \text { if } 0 \leq \mu<\bar{\mu}-1, \\
o_{1}\left(\varepsilon^{2}|\log \varepsilon|\right) & \text { if } \mu=\bar{\mu}-1,\end{cases}
\end{gathered}
$$

where $C_{\mu}=\left(\frac{4 N(\bar{\mu}-\mu)}{N-2}\right)^{\frac{N-2}{4}}, \omega_{N}$ is the volume of the unit ball in $\mathbb{R}^{N}$. 


\section{Asymptotic behavior of solutions}

Proof of Theorem 1.1 Suppose $\left(u_{0}, v_{0}\right) \in H \times H$ is a nontrivial solution to problem (1.1). For all $0 \leq \mu_{i} \leq \bar{\mu}$ define

$$
u(x)=\left|x-\xi_{i}\right|^{\gamma} u_{0}(x) \quad \text { and } \quad v(x)=\left|x-\xi_{i}\right|^{\gamma} \nu_{0}(x) \text {, where } \gamma=\left(\sqrt{\bar{\mu}}-\sqrt{\bar{\mu}-\mu_{i}}\right) \text {. }
$$

It is not difficult to verify that $u, v \in H_{0}^{1}\left(\Omega,\left|x-\xi_{i}\right|^{-2 \gamma}\right)$ and satisfy

$$
\left\{\begin{array}{l}
-\operatorname{div}\left(\left|x-\xi_{i}\right|^{-2 \gamma} \nabla u\right) \\
=\frac{\sigma \alpha}{2^{*}}\left|x-\xi_{i}\right|^{-2^{*} \gamma} u|u|^{\alpha-2}|\nu|^{\beta}+\eta\left|x-\xi_{i}\right|^{-2^{*} \gamma} u|u|^{2^{*}-2} \\
\quad+a_{1}\left|x-\xi_{i}\right|^{-2 \gamma} u+a_{2}\left|x-\xi_{i}\right|^{-2 \gamma} \nu+\sum_{\substack{j=1 \\
j \neq i}}^{k} \frac{\mu_{j}}{\left|x-\xi_{j}\right|^{2}}\left|x-\xi_{i}\right|^{-2 \gamma} u \\
-\operatorname{div}\left(\left|x-\xi_{i}\right|^{-2 \gamma} \nabla v\right) \\
\quad=\frac{\sigma \beta}{2^{*}}\left|x-\xi_{i}\right|^{-2^{*} \gamma} \nu|\nu|^{\beta-2}|u|^{\alpha}+\lambda\left|x-\xi_{i}\right|^{-2^{*} \gamma} \nu|\nu|^{2^{*}-2} \\
\quad+a_{2}\left|x-\xi_{i}\right|^{-2 \gamma} u+a_{3}\left|x-\xi_{i}\right|^{-2 \gamma} \nu+\sum_{\substack{j=1 \\
j \neq i}}^{k} \frac{\mu_{j}}{\left|x-\xi_{j}\right|^{2}}\left|x-\xi_{i}\right|^{-2 \gamma} \nu .
\end{array}\right.
$$

Let $R>0$ small enough such that $B_{R}\left(\xi_{i}\right) \subset \Omega$ and $\xi_{i} \notin B_{R}\left(\xi_{i}\right)$ for $j \neq i$. Also, let $\varphi_{i} \in$ $C_{0}^{\infty}\left(B_{R}\left(\xi_{i}\right)\right)$ be a cut-off function. Set

$$
u_{n}:=\min \{|u|, n\} ; \quad v_{n}:=\min \{|v|, n\} ; \quad \phi_{1_{i}}:=\varphi_{i}^{2} u u_{n}^{2(s-1)}, \quad \phi_{2_{i}}:=\varphi_{i}^{2} \nu v_{n}^{2(s-1)},
$$

where $s, n>1$. Multiplying the first equation of (3.1) by $\phi_{1_{i}}$ and the second one by $\phi_{2_{i}}$ respectively and integrating, we have

$$
\begin{aligned}
\int_{\Omega}\left|x-\xi_{i}\right|^{-2 \gamma} \nabla u \nabla \phi_{1_{i}}= & \frac{\sigma \alpha}{2^{*}} \int_{\Omega}\left|x-\xi_{i}\right|^{-2^{*} \gamma} u|u|^{\alpha-2}|\nu|^{\beta} \phi_{1_{i}}+\eta \int_{\Omega}\left|x-\xi_{i}\right|^{-2^{*} \gamma} \\
& \times u|u|^{2^{*}-2} \phi_{1_{i}}+a_{1} \int_{\Omega}\left|x-\xi_{i}\right|^{-2 \gamma} u \phi_{1_{i}}+a_{2} \int_{\Omega}\left|x-\xi_{i}\right|^{-2 \gamma} \\
& \times v \phi_{1_{i}}+\sum_{\substack{j=1 \\
j \neq i}}^{k} \frac{\mu_{j}}{\left|x-\xi_{j}\right|^{2}}\left|x-\xi_{i}\right|^{-2 \gamma} u \phi_{1_{i}} .
\end{aligned}
$$

Note that $\nabla \phi_{1_{i}}=2 \varphi_{i} u u_{n}^{2(s-1)} \nabla \varphi_{i}+\varphi_{i}^{2} u_{n}^{2(s-1)} \nabla u+2(s-1) \varphi_{i}^{2} u_{n}^{2(s-1)} \nabla u_{n}$.

Then

$$
\begin{aligned}
\int_{\Omega}\left|x-\xi_{i}\right|^{-2 \gamma} \nabla u \nabla \phi_{1_{i}}= & 2 \int_{\Omega}\left|x-\xi_{i}\right|^{-2 \gamma} \varphi_{i} u u_{n}^{2(s-1)} \nabla \varphi_{i} \nabla u+\int_{\Omega}\left|x-\xi_{i}\right|^{-2 \gamma} \varphi_{i}^{2} \\
& \times u_{n}^{2(s-1)}|\nabla u|^{2}+2(s-1) \int_{\Omega}\left|x-\xi_{i}\right|^{-2 \gamma} \varphi_{i}^{2} u_{n}^{2(s-1)}\left|\nabla u_{n}\right|^{2} .
\end{aligned}
$$

By the Cauchy inequality and the Young inequality, we get

$$
\begin{aligned}
& \left|2 \int_{\Omega}\right| x-\left.\xi_{i}\right|^{-2 \gamma} \varphi_{i} u u_{n}^{2(s-1)} \nabla \varphi_{i} \nabla u \mid \\
& \quad \leq \frac{1}{2} \int_{\Omega}\left|x-\xi_{i}\right|^{-2 \gamma} \varphi_{i}^{2} u_{n}^{2(s-1)}|\nabla u|^{2}+2 \int_{\Omega}\left|x-\xi_{i}\right|^{-2 \gamma}\left|\nabla \varphi_{i}\right|^{2} u^{2} u_{n}^{2(s-1)} .
\end{aligned}
$$


The same result holds for $\int_{\Omega}\left|x-\xi_{i}\right|^{-2 \gamma} \nabla \phi_{2_{i}} \nabla v$.

By letting $\psi_{1}(x)=\varphi_{i} u u_{n}^{(s-1)}, \psi_{2}(x)=\varphi_{i} \nu v_{n}^{(s-1)}$, we have

$$
\begin{aligned}
\int_{\Omega} \mid x & -\left.\xi_{i}\right|^{-2 \gamma}\left(\left|\nabla \psi_{1}\right|^{2}+\left|\nabla \psi_{2}\right|^{2}\right) \\
\leq & C\left(\int_{\Omega}\left|x-\xi_{i}\right|^{-2 \gamma}\left|\nabla \varphi_{i}\right|^{2} u^{2} u_{n}^{2(s-1)}+\int_{\Omega}\left|x-\xi_{i}\right|^{-2 \gamma} \nabla u \nabla \phi_{1_{i}}\right. \\
& \left.+\int_{\Omega}\left|x-\xi_{i}\right|^{-2 \gamma}\left|\nabla \varphi_{i}\right|^{2} v^{2} v_{n}^{2(s-1)}+\int_{\Omega}\left|x-\xi_{i}\right|^{-2 \gamma} \nabla v \nabla \phi_{2_{i}}\right) \\
\leq & C\left(\int_{\Omega}\left|x-\xi_{i}\right|^{-2 \gamma}\left|\nabla \varphi_{i}\right|^{2}\left(u^{2} u_{n}^{2(s-1)}+v^{2} v_{n}^{2(s-1)}\right)\right. \\
& +\int_{\Omega}\left|x-\xi_{i}\right|^{-2^{*} \gamma} \varphi_{i}^{2}|u|^{\alpha}|v|^{\beta}\left(u_{n}^{2(s-1)}+v_{n}^{2(s-1)}\right) \\
& +\int_{\Omega}\left|x-\xi_{i}\right|^{-2^{*} \gamma} \varphi_{i}^{2}|u|^{2^{*}} u_{n}^{2(s-1)}+\int_{\Omega}\left|x-\xi_{i}\right|^{-2^{*} \gamma} \varphi_{i}^{2}|v|^{2^{*}} v_{n}^{2(s-1)} \\
& +\sum_{\substack{j=1 \\
j \neq i}}^{k} \mu_{j} \int_{\Omega} \frac{\left|x-\xi_{i}\right|^{-2 \gamma}}{\left|x-\xi_{j}\right|^{2}} \varphi_{i}^{2}\left(u^{2} u_{n}^{2(s-1)}+v^{2} v_{n}^{2(s-1)}\right) \\
& \left.+\int_{\Omega}\left|x-\xi_{i}\right|^{-2 \gamma} \varphi_{i}^{2}\left(u^{2}+v^{2}\right)\left(u_{n}^{2(s-1)}+v_{n}^{2(s-1)}\right)\right) .
\end{aligned}
$$

Using Caffarelli-Kohn-Nirenberg inequality [10], we infer that

$$
\begin{aligned}
& \left(\int_{\Omega} \frac{\left|\varphi_{i} u u_{n}^{(s-1)}\right|^{*^{*}}}{\left|x-\xi_{i}\right|^{2^{*} \gamma}}\right)^{\frac{2}{2^{*}}}+\left(\int_{\Omega} \frac{\left|\varphi_{i} v v_{n}^{(s-1)}\right|^{2^{*}}}{\left|x-\xi_{i}\right|^{2^{*} \gamma}}\right)^{\frac{2}{2^{*}}} \\
& \leq C\left(\int_{\Omega}\left|x-\xi_{i}\right|^{-2 \gamma}\left|\nabla\left(\varphi_{i} u u_{n}^{(s-1)}\right)\right|^{2}+\int_{\Omega}\left|x-\xi_{i}\right|^{-2 \gamma}\left|\nabla\left(\varphi_{i} v v_{n}^{(s-1)}\right)\right|^{2}\right) \\
& =C \int_{\Omega}\left|x-\xi_{i}\right|^{-2 \gamma}\left(\left|\nabla \psi_{1}\right|^{2}+\left|\nabla \psi_{2}\right|^{2}\right) \\
& \leq C\left(\int_{\Omega}\left|x-\xi_{i}\right|^{-2 \gamma}\left|\nabla \varphi_{i}\right|^{2}\left(u^{2} u_{n}^{2(s-1)}+v^{2} v_{n}^{2(s-1)}\right)\right. \\
& \quad+\int_{\Omega}\left|x-\xi_{i}\right|^{-2^{*} \gamma} \varphi_{i}^{2}|u|^{\alpha}|v|^{\beta}\left(u_{n}^{2(s-1)}+v_{n}^{2(s-1)}\right) \\
& \quad+\int_{\Omega}\left|x-\xi_{i}\right|^{-2^{*} \gamma} \varphi_{i}^{2}|u|^{2^{*}} u_{n}^{2(s-1)}+\int_{\Omega}\left|x-\xi_{i}\right|^{-2^{*} \gamma} \varphi_{i}^{2}|v|^{2^{*}} v_{n}^{2(s-1)} \\
& \quad+\sum_{\substack{j=1 \\
j \neq i}}^{k} \mu_{j} \int_{\Omega} \frac{\left|x-\xi_{i}\right|^{-2 \gamma}}{\left|x-\xi_{j}\right|^{2}} \varphi_{i}^{2}\left(u^{2} u_{n}^{2(s-1)}+v^{2} v_{n}^{2(s-1)}\right) \\
& \left.\quad+\int_{\Omega}\left|x-\xi_{i}\right|^{-2 \gamma} \varphi_{i}^{2}\left(u^{2}+v^{2}\right)\left(u_{n}^{2(s-1)}+v_{n}^{2(s-1)}\right)\right) .
\end{aligned}
$$

Define

$$
\omega(x):=\max \{u(x), v(x)\}, \quad \omega_{n}(x):=\min \{\omega(x), n\} .
$$


Then $\omega_{n}(x):=\max \left\{u_{n}(x), v_{n}(x)\right\}$. Now, from the Hölder inequality, we deduce that

$$
\begin{aligned}
& \int_{\Omega} \frac{\varphi_{i}^{2}|\omega|^{2^{*}} \omega_{n}^{2(s-1)}}{\left|x-\xi_{i}\right|^{2^{*} \gamma}} \leq\left(\int_{\Omega} \frac{\left|\varphi_{i} \omega \omega_{n}^{(s-1)}\right|^{2^{*}}}{\left|x-\xi_{i}\right|^{2^{*} \gamma}}\right)^{\frac{2}{2^{*}}}\left(\int_{B_{R}\left(\xi_{i}\right)} \frac{|\omega|^{2^{*}}}{\left|x-\xi_{i}\right|^{2^{*} \gamma}}\right)^{\frac{2}{N}}, \\
& \int_{\Omega} \frac{\varphi_{i}^{2}|\omega|^{2} \omega_{n}^{2(s-1)}}{\left|x-\xi_{i}\right|^{2 \gamma}} \leq\left(\int_{\Omega} \frac{\left|\varphi_{i} \omega \omega_{n}^{(s-1)}\right|^{2^{*}}}{\left|x-\xi_{i}\right|^{2^{*} \gamma}}\right)^{\frac{2}{2^{*}}}\left|B_{R}\left(\xi_{i}\right)\right|^{\frac{2}{N}}
\end{aligned}
$$

In the sequel, we have

$$
\begin{aligned}
& \int_{\Omega}\left|x-\xi_{i}\right|^{-2^{*} \gamma} \varphi_{i}^{2}|u|^{\alpha}|\nu|^{\beta}\left(u_{n}^{2(s-1)}+v_{n}^{2(s-1)}\right) \\
& \quad \leq \int_{\Omega}\left|x-\xi_{i}\right|^{-2^{*} \gamma} \varphi_{i}^{2}|\omega|^{\alpha}|\omega|^{\beta}\left(\omega_{n}^{2(s-1)}+\omega_{n}^{2(s-1)}\right) \\
& =\int_{\Omega}\left|x-\xi_{i}\right|^{-2^{*} \gamma} \varphi_{i}^{2}|\omega|^{2^{*}} \omega_{n}^{2(s-1)} \\
& \quad \leq 2\left(\int_{\Omega} \frac{\left|\varphi_{i} \omega \omega_{n}^{(s-1)}\right|^{2^{*}}}{\left|x-\xi_{i}\right|^{2^{*} \gamma}}\right)^{\frac{2}{2^{*}}}\left(\int_{B_{R}\left(\xi_{i}\right)} \frac{|\omega|^{2^{*}}}{\left|x-\xi_{i}\right|^{2^{*} \gamma}}\right)^{\frac{2}{N}} .
\end{aligned}
$$

By the choice of $\varphi_{i}$, we obtain

$$
\begin{aligned}
& \sum_{\substack{j=1 \\
j \neq i}}^{k} \mu_{j} \int_{\Omega} \frac{\left|x-\xi_{i}\right|^{-2 \gamma}}{\left|x-\xi_{j}\right|^{2}} \varphi_{i}^{2}\left(u^{2} u_{n}^{2(s-1)}+v^{2} v_{n}^{2(s-1)}\right) \\
& \leq C \int_{\Omega}\left|x-\xi_{i}\right|^{-2 \gamma} \varphi_{i}^{2}\left(u^{2} u_{n}^{2(s-1)}+v^{2} v_{n}^{2(s-1)}\right) \\
& \quad \leq \int_{\Omega}\left|x-\xi_{i}\right|^{-2 \gamma} \varphi_{i}^{2} \omega^{2} \omega_{n}^{2(s-1)} \\
& \quad \leq 2 C\left(\int_{\Omega} \frac{\left|\varphi_{i} \omega \omega_{n}^{(s-1)}\right|^{2^{*}}}{\left|x-\xi_{i}\right|^{2^{*} \gamma}}\right)^{\frac{2}{2^{*}}}\left|B_{R}\left(\xi_{i}\right)\right|^{\frac{2}{N}} .
\end{aligned}
$$

So, from (3.4) to (3.8) it follows that

$$
\begin{aligned}
& \left(\int_{\Omega}\left|x-\xi_{i}\right|^{-2^{*} \gamma}\left|\varphi_{i} \omega \omega_{n}^{s-1}\right|^{2^{*}}\right)^{\frac{2}{2^{*}}} \\
& \quad \leq C \int_{\Omega}\left|x-\xi_{i}\right|^{-2 \gamma}\left|\nabla \varphi_{i}\right|^{2} \omega^{2} \omega_{n}^{2(s-1)} .
\end{aligned}
$$

Take $s=\frac{2^{*}}{2}$ and $\varphi_{i}(x)$ to be a constant near the zero. Letting $n \rightarrow \infty$, we infer that $\omega \in$ $L^{\frac{2^{*^{2}}}{2}}\left(B_{R}\left(\xi_{i}\right),\left|x-\xi_{i}\right|^{-2^{*} \gamma}\right)$ and so

$$
u, v \in L^{\frac{2^{*^{2}}}{2}}\left(B_{R}\left(\xi_{i}\right),\left|x-\xi_{i}\right|^{-2^{*} \gamma}\right)
$$

Suppose $r>0$ is sufficiently small such that $r+l<R$ and $\varphi_{i}$ is a cut-off function with the properties $\left|\nabla \varphi_{i}\right|<\frac{1}{l}$ and $\varphi_{i}(x)=1$ in $B_{r}\left(\xi_{i}\right)$.

Set $t:=\frac{2^{*^{2}}}{2\left(2^{*}-2\right)}, \delta:=2 \gamma t-2^{*} \gamma(t-1)$. 
Then we have the following results:

$$
\begin{aligned}
& \int_{\Omega}\left|x-\xi_{i}\right|^{-2 \gamma}\left|\nabla \varphi_{i}\right|^{2} \omega^{2} \omega_{n}^{2(s-1)} \\
& \leq C l^{-2} \int_{\Omega}\left|x-\xi_{i}\right|^{-\left(2^{*} \gamma \frac{t-1}{t}\right)} \omega^{2} \omega_{n}^{2(s-1)}\left|x-\xi_{i}\right|^{-\gamma\left(2-2^{*} \frac{t-1}{t}\right)} \\
& \leq C l^{-2}\left(\int_{B_{r+l}\left(\xi_{i}\right)} \frac{\left|\omega \omega_{n}^{(s-1)}\right|^{\frac{2 t}{t-1}}}{\left|x-\xi_{i}\right|^{2^{*} \gamma}}\right)^{\frac{t-1}{t}}\left(\int_{B_{r+l}\left(\xi_{i}\right)} \frac{1}{\left|x-\xi_{i}\right|^{\delta}}\right)^{\frac{1}{t}} \\
& \leq C l^{-2}\left(\int_{B_{r+l}\left(\xi_{i}\right)} \frac{\left|\omega \omega_{n}^{(s-1)}\right| \frac{2 t}{t-1}}{\left|x-\xi_{i}\right|^{2^{*} \gamma}}\right)^{\frac{t-1}{t}},
\end{aligned}
$$

where we used the Hölder inequality. From (3.9) in combination with (3.11), it follows that

$$
\left(\int_{B_{r+l}\left(\xi_{i}\right)}\left|x-\xi_{i}\right|^{2^{*} \gamma}|\omega|^{2^{*} s}\right)^{\frac{1}{2^{*} s}} \leq C^{\frac{1}{2 s}} l^{-\frac{1}{2 s}}\left(\int_{B_{r+l}\left(\xi_{i}\right)}\left|x-\xi_{i}\right|^{2^{*} \gamma}|\omega|^{\bar{p}_{0} s}\right)^{\frac{1}{p_{0} s}}
$$

where $\bar{p}_{0}=\frac{2 t}{t-1}<2^{*}$.

Denote $s=\chi^{j}, \chi=\frac{p}{\bar{p}_{0}}$ and $l=\rho^{j}, j \geq 1$, where $\chi \geq 1,2^{*} \gamma<N$ and $\bar{p}_{0} \chi^{j}=p \chi^{j-1}$. Using (3.12) recursively, we get

$$
\begin{aligned}
\left(\int_{B_{r}\left(\xi_{i}\right)}|\omega|^{2^{*} \chi^{j}}\right)^{\frac{1}{2^{*} \chi^{j}}} & \leq r x^{\frac{\gamma}{x^{j}}}\left(\int_{B_{r}\left(\xi_{i}\right)} \frac{|\omega|^{2^{*} \chi^{j}}}{\left|x-\xi_{i}\right|^{2^{*} \gamma}}\right)^{\frac{1}{2^{*} \chi^{j}}} \\
& \leq r \chi^{\frac{\gamma}{j}} C^{\sum_{i=1}^{j} \frac{1}{2 \chi^{j}}} \rho^{-\sum_{i=1}^{j} \frac{i}{\chi^{i}}}\left(\int_{B_{r+\rho}\left(\xi_{i}\right)}\left|x-\xi_{i}\right|^{2^{*} \gamma}|\omega|^{2^{*}}\right)^{\frac{1}{2^{*}}}
\end{aligned}
$$

we have $\chi^{j} \rightarrow \infty$ as $j \rightarrow \infty$. Note that the infinite sums on the right-hand side converge, then we obtain that $\omega \in L^{\infty}\left(B_{r}\left(\xi_{i}\right)\right)$, particularly, we have $u, v \in L^{\infty}(\Omega)$. Thus,

$$
u_{0}(x)=\left|x-\xi_{i}\right|^{-\gamma} u(x) \leq M_{1}\left|x-\xi_{i}\right|^{-\gamma} \quad \text { for } x \in B_{r}\left(\xi_{i}\right) \backslash\left\{\xi_{i}\right\}
$$

where $M_{1}=\max \left\{\|u\|_{L^{\infty}\left(B_{r}\left(\xi_{i}\right)\right)}, 1 \leq i \leq k\right\}$

$$
\nu_{0}(x)=\left|x-\xi_{i}\right|^{-\gamma} \nu(x) \leq M_{2}\left|x-\xi_{i}\right|^{-\gamma} \quad \text { for } x \in B_{r}\left(\xi_{i}\right) \backslash\left\{\xi_{i}\right\},
$$

where $M_{1}=\max \left\{\|v\|_{L^{\infty}\left(B_{r}\left(\xi_{i}\right)\right)}, 1 \leq i \leq k\right\}$. The proof is complete.

Proof of Theorem 1.2 Suppose $\left(u_{0}, v_{0}\right) \in H \times H$ is a positive solution to problem (1.1). For all $0 \leq \mu_{i} \leq \bar{\mu}$, set

$$
u(x)=\left|x-\xi_{i}\right|^{\gamma} u_{0}(x) \quad \text { and } \quad v(x)=\left|x-\xi_{i}\right|^{\gamma} v_{0}(x) .
$$


Then

$$
\left\{\begin{array}{l}
-\operatorname{div}\left(\left|x-\xi_{i}\right|^{-2 \gamma} \nabla u\right) \\
=\frac{\sigma \alpha}{2^{*}}\left|x-\xi_{i}\right|^{-2^{*} \gamma} u^{\alpha-1} v^{\beta}+\eta\left|x-\xi_{i}\right|^{-2^{*} \gamma} u^{2^{*}-1} \\
\quad+a_{1}\left|x-\xi_{i}\right|^{-2 \gamma} u+a_{2}\left|x-\xi_{i}\right|^{-2 \gamma} v+\sum_{\substack{j=1 \\
j \neq i}}^{k} \frac{\mu_{j}}{\left|x-\xi_{j}\right|^{2}}\left|x-\xi_{i}\right|^{-2 \gamma} u \\
-\operatorname{div}\left(\left|x-\xi_{i}\right|^{-2 \gamma} \nabla v\right) \\
=\frac{\sigma \beta}{2^{*}}\left|x-\xi_{i}\right|^{-2^{*} \gamma} \nu^{\beta-1} u^{\alpha}+\lambda\left|x-\xi_{i}\right|^{-2^{*} \gamma} v^{2^{*}-1} \\
\quad+a_{2}\left|x-\xi_{i}\right|^{-2 \gamma} u+a_{3}\left|x-\xi_{i}\right|^{-2 \gamma} v+\sum_{\substack{j=1 \\
j \neq i}}^{k} \frac{\mu_{j}}{\left|x-\xi_{j}\right|^{2}}\left|x-\xi_{i}\right|^{-2 \gamma} v .
\end{array}\right.
$$

Choose $0<\rho_{0}<\rho$ and define $n(t)=\min _{\left|x-\xi_{i}\right|=t} u(x)$ for $\rho_{0} \leq t \leq \rho$. Let

$$
\begin{aligned}
& n\left(\rho_{0}\right)=A \rho_{0}^{-2 \sqrt{\mu-\mu_{i}}}+B, \quad n(\rho)=A \rho^{-2 \sqrt{\bar{\mu}-\mu_{i}}}+B, \quad \text { where } \\
& A=\frac{n(\rho)-n\left(\rho_{0}\right)}{\rho^{-2 \sqrt{\bar{\mu}-\mu_{i}}}-\rho_{0}^{-2 \sqrt{\bar{\mu}-\mu_{i}}},} \quad B=\frac{n\left(\rho_{0}\right) \rho^{-2 \sqrt{\bar{\mu}-\mu_{i}}}-n(\rho) \rho_{0}^{-2 \sqrt{\bar{\mu}-\mu_{i}}}}{\rho^{-2 \sqrt{\bar{\mu}-\mu_{i}}}-\rho_{0}^{-2 \sqrt{\bar{\mu}-\mu_{i}}}} .
\end{aligned}
$$

It is easy to verify that

$$
-\operatorname{div}\left(\left|x-\xi_{i}\right|^{-2\left(\sqrt{\bar{\mu}}-\sqrt{\bar{\mu}-\mu_{i}}\right)} \nabla\left(A\left|x-\xi_{i}\right|^{-2 \sqrt{\bar{\mu}-\mu_{i}}}+B\right)\right)=0 \quad \forall x \in \Omega \backslash\left\{\xi_{i}\right\} .
$$

Combining (3.13) with (3.14), we get

$$
\begin{aligned}
& -\operatorname{div}\left(\left|x-\xi_{i}\right|^{-2\left(\sqrt{\bar{\mu}}-\sqrt{\bar{\mu}-\mu_{i}}\right)} \nabla\left(u-A\left|x-\xi_{i}\right|^{-2 \sqrt{\bar{\mu}-\mu_{i}}}+B\right)\right) \geq 0, \quad \forall x \in B_{\rho}\left(\xi_{i}\right) \backslash B_{\rho_{0}}\left(\xi_{i}\right), \\
& u(x)-\left(A\left|x-\xi_{i}\right|^{-2 \sqrt{\bar{\mu}-\mu_{i}}}+B\right) \geq 0, \quad \forall x \in \partial\left(B_{\rho}\left(\xi_{i}\right) \backslash B_{\rho_{0}}\left(\xi_{i}\right)\right) .
\end{aligned}
$$

Therefore, by the maximum principle in $B_{\rho}\left(\xi_{i}\right) \backslash B_{\rho_{0}}\left(\xi_{i}\right)$, we obtain

$$
u(x)-\left(A\left|x-\xi_{i}\right|^{-2 \sqrt{\mu-\mu_{i}}}+B\right) \geq 0, \quad \forall x \in B_{\rho}\left(\xi_{i}\right) \backslash B_{\rho_{0}}\left(\xi_{i}\right) .
$$

Thus, for all $x \in B_{\rho}\left(\xi_{i}\right) \backslash B_{\rho_{0}}\left(\xi_{i}\right)$,

$$
\begin{aligned}
& u(x) \geq A\left|x-\xi_{i}\right|^{-2 \sqrt{\bar{\mu}-\mu_{i}}}+B \\
& =\frac{\left|x-\xi_{i}\right|^{-2 \sqrt{\bar{\mu}-\mu_{i}}}-\rho^{-2 \sqrt{\bar{\mu}-\mu_{i}}}}{\rho_{0}^{-2 \sqrt{\bar{\mu}-\mu_{i}}}-\rho^{-2 \sqrt{\mu-\mu_{i}}}} n\left(\rho_{0}\right)+\frac{\rho_{0}^{-2 \sqrt{\bar{\mu}-\mu_{i}}}-\left|x-\xi_{i}\right|^{-2 \sqrt{\bar{\mu}-\mu_{i}}}}{\rho_{0}^{-2 \sqrt{\bar{\mu}-\mu_{i}}}-\rho^{-2 \sqrt{\mu-\mu_{i}}}} n(\rho) \\
& \geq \frac{\left|x-\xi_{i}\right|^{2 \sqrt{\bar{\mu}-\mu_{i}}}-\rho_{0}^{2 \sqrt{\mu-\mu_{i}}}}{\left|x-\xi_{i}\right|^{2 \sqrt{\bar{\mu}-\mu_{i}}}-\rho_{0}^{2 \sqrt{\mu-\mu_{i}}} \rho^{-2 \sqrt{\bar{\mu}-\mu_{i}}}\left|x-\xi_{i}\right|^{2 \sqrt{\bar{\mu}-\mu_{i}}}} n(\rho) .
\end{aligned}
$$

Taking $\rho_{0} \rightarrow 0$, we conclude $u(x) \geq n(\rho)=\min _{\left|x-\xi_{i}\right|=\rho} u(x)>0$ for all $x \in B_{\rho}\left(\xi_{i}\right) \backslash\left\{\xi_{i}\right\}$.

Similar result also holds for $v(x)$. Therefore, we have

$$
\begin{aligned}
u_{0}(x) & =\left|x-\xi_{i}\right|^{-\gamma} u(x) \geq\left|x-\xi_{i}\right|^{-\gamma} \min _{\left|x-\xi_{i}\right|=\rho} u(x)=\left|x-\xi_{i}\right|^{-\gamma} C_{i} \\
& \geq\left|x-\xi_{i}\right|^{-\gamma} \min _{i=1,2, \ldots, k} C_{i}=\left|x-\xi_{i}\right|^{-\gamma} N_{1} .
\end{aligned}
$$


For any $x \in B_{\rho}\left(\xi_{i}\right) \backslash\left\{\xi_{i}\right\}$,

$$
\begin{aligned}
\nu_{0}(x) & =\left|x-\xi_{i}\right|^{-\gamma} \nu(x) \geq\left|x-\xi_{i}\right|^{-\gamma} \min _{\left|x-\xi_{i}\right|=\rho} v(x)=\left|x-\xi_{i}\right|^{-\gamma} \dot{C}_{i} \\
& \geq\left|x-\xi_{i}\right|^{-\gamma} \min _{i=1,2, \ldots, k} \dot{C}_{i}=\left|x-\xi_{i}\right|^{-\gamma} N_{2} .
\end{aligned}
$$

For any $x \in B_{\rho}\left(\xi_{i}\right) \backslash\left\{\xi_{i}\right\}$. This proves the theorem.

\section{Local $(P S)_{c}$-condition and the existence of positive solutions}

We first establish a compactness result.

Lemma 4.1 Suppose that $\left(\mathcal{H}_{1}\right)$ holds. Then J satisfies the $(P S)_{c}$-condition for all

$$
c<c^{*}:=\frac{1}{N} \min \left\{S_{\eta, \lambda, \sigma}^{\frac{N}{2}}\left(\mu_{1}\right), \ldots, S_{\eta, \lambda, \sigma}^{\frac{N}{2}}\left(\mu_{k}\right),\left(S_{0}\right)^{\frac{N}{2}}\right\}=\frac{1}{N} S_{\eta, \lambda, \sigma}^{\frac{N}{2}}\left(\mu_{k}\right) .
$$

Proof Suppose that $\left\{\left(u_{n}, v_{n}\right)\right\} \subset H \times H$ satisfies $J\left(u_{n}, v_{n}\right) \rightarrow c<c^{*}$ and $J^{\prime}\left(u_{n}, v_{n}\right) \rightarrow 0$. The standard argument shows that $\left\{\left(u_{n}, v_{n}\right)\right\}$ is bounded in $H \times H$.

For some $(u, v) \in H \times H$, we have

$$
\begin{aligned}
& \left(u_{n}, v_{n}\right) \rightarrow(u, v) \quad \text { weakly in } H \times H, \\
& \left(u_{n}, v_{n}\right) \rightarrow(u, v) \quad \text { weakly in } L^{2}\left(\Omega,\left|x-\xi_{i}\right|^{-2}\right) \times L^{2}\left(\Omega,\left|x-\xi_{i}\right|^{-2}\right), \\
& \left(u_{n}, v_{n}\right) \rightarrow(u, v) \quad \text { weakly in } L^{2^{*}}(\Omega) \times L^{2^{*}}(\Omega), \\
& \left(u_{n}, v_{n}\right) \rightarrow(u, v) \quad \text { strongly in } L^{q_{1}}(\Omega) \times L^{q_{2}}(\Omega), q_{1}, q_{2} \in\left[1,2^{*}\right), \\
& \left(u_{n}, v_{n}\right) \rightarrow(u, v) \text { a.e. in } \Omega .
\end{aligned}
$$

Therefore, $(u, v)$ is a solution to (1.1). Then by the concentration-compactness principle [11-13] and up to a subsequence, there exist an at most countable set $\mathcal{J}$, a set of different points $\left\{x_{j}\right\}_{j \in \mathcal{J}} \subset \Omega \backslash \xi_{i=1}^{k}$, nonnegative real numbers $\tilde{\tau}_{x_{j}}, \tilde{v}_{x_{j}}, j \in \mathcal{J}$, and $\tilde{\tau}_{\xi_{i}}, \tilde{v}_{\xi_{i}}, \tilde{\gamma}_{\xi_{i}}(1 \leq i \leq k)$ such that the following convergence holds in the sense of measures:

$$
\begin{aligned}
& \left|\nabla u_{n}\right|^{2}+\left|\nabla v_{n}\right|^{2} \rightarrow d \tilde{\tau} \geq|\nabla u|^{2}+|\nabla v|^{2}+\sum_{j \in \mathcal{J}} \tilde{\tau}_{x_{j}} \delta_{x_{j}}+\sum_{i=1}^{k} \tilde{\tau}_{\xi_{i}} \delta_{\xi_{i}}, \\
& \frac{u_{n}^{2}+v_{n}^{2}}{\left|x-\xi_{i}\right|^{2}} \rightarrow d \tilde{\gamma}=\frac{u^{2}+v^{2}}{\left|x-\xi_{i}\right|^{2}}+\tilde{\gamma}_{\xi_{i}} \delta_{\xi_{i}}, \\
& \eta\left|u_{n}\right|^{2^{*}}+\lambda\left|v_{n}\right|^{2^{*}}+\sigma\left|u_{n}\right|^{\alpha}\left|\nu_{n}\right|^{\beta} \rightarrow d \tilde{v} \\
& =\eta|u|^{2^{*}}+\lambda|\nu|^{2^{*}} \\
& \quad+\sigma|u|^{\alpha}|\nu|^{\beta}+\sum_{j \in \mathcal{J}} \tilde{v}_{x_{j}} \delta_{x_{j}}+\sum_{i=1}^{k} \tilde{\nu}_{\xi_{i}} \delta_{\xi_{i}} .
\end{aligned}
$$

By the Sobolev inequalities [10], we have

$$
S_{\mu_{i}} \tilde{\nu}_{\xi_{i}}^{\frac{2}{2 *}} \leq \tilde{\tau}_{\xi_{i}}-\mu_{i} \tilde{\gamma}_{\xi_{i}}, \quad 1 \leq i \leq k
$$


We claim that $\mathcal{J}$ is finite, and for any $j \in \mathcal{J}, \tilde{v}_{x_{j}}=0$ or $\tilde{v}_{x_{j}} \geq S_{0} \frac{N}{2}$.

In fact, let $\varepsilon>0$ be small enough for any $1 \leq i \leq k, \xi_{i} \notin B_{\varepsilon}\left(x_{j}\right)$ and $B_{\varepsilon}\left(x_{i}\right) \cap B_{\varepsilon}\left(x_{j}\right)=\emptyset$ for $i \neq j, i, j \in \mathcal{J}$. Let $\phi_{\varepsilon}^{j}$ be a smooth cut-off function centered at $x_{j}$ such that $0 \leq \phi_{\varepsilon}^{j} \leq 1, \phi_{\varepsilon}^{j}=1$ for $\left|x-x_{j}\right| \leq \frac{\varepsilon}{2}, \phi_{\varepsilon}^{j}=0$ for $\left|x-x_{j}\right| \geq \varepsilon$ and $\left|\nabla \phi_{\varepsilon}^{j}\right| \leq \frac{4}{\varepsilon}$. Then

$$
\begin{aligned}
& \lim _{\varepsilon \rightarrow 0} \lim _{n \rightarrow \infty} \int_{\Omega}\left(\left|\nabla u_{n}\right|^{2}+\left|\nabla v_{n}\right|^{2}\right) \phi_{\varepsilon}^{j}=\lim _{\varepsilon \rightarrow 0} \int_{\Omega} \phi_{\varepsilon}^{j} d \tilde{\tau} \\
& \geq \lim _{\varepsilon \rightarrow 0}\left(\int_{\Omega}\left(|\nabla u|^{2}+|\nabla v|^{2}\right) \phi_{\varepsilon}^{j}+\tilde{\tau}_{x_{j}}\right) \\
& =\tilde{\tau}_{x_{j}}, \\
& \lim _{\varepsilon \rightarrow 0} \lim _{n \rightarrow \infty} \int_{\Omega} \frac{u_{n}^{2}+v_{n}^{2}}{\left|x-\xi_{i}\right|^{2}} \phi_{\varepsilon}^{j}=\lim _{\varepsilon \rightarrow 0} \int_{\Omega} \phi_{\varepsilon}^{j} d \tilde{\gamma}=\int_{\Omega} \frac{u^{2}+v^{2}}{\left|x-\xi_{i}\right|^{2}} \phi_{\varepsilon}^{j}=0, \\
& \lim _{\varepsilon \rightarrow 0} \lim _{n \rightarrow \infty} \int_{\Omega}\left(\eta\left|u_{n}\right|^{2^{*}}+\lambda\left|v_{n}\right|^{2^{*}}+\sigma\left|u_{n}\right|^{\alpha}\left|v_{n}\right|^{\beta}\right) \phi_{\varepsilon}^{j} \\
& =\lim _{\varepsilon \rightarrow 0} \int_{\Omega} \phi_{\varepsilon}^{j} d \tilde{v} \\
& =\lim _{\varepsilon \rightarrow 0}\left(\int_{\Omega}\left(\eta|u|^{2^{*}}+\lambda|v|^{2^{*}}+\sigma|u|^{\alpha}|v|^{\beta}\right) \phi_{\varepsilon}^{j}+\tilde{v}_{x_{j}}\right) \\
& =\tilde{v}_{x_{j}} \text {, } \\
& \lim _{\varepsilon \rightarrow 0} \lim _{n \rightarrow \infty} \int_{\Omega}\left(u_{n} \nabla u_{n}+v_{n} \nabla v_{n}\right) \nabla \phi_{\varepsilon}^{j}=0 .
\end{aligned}
$$

Then we have

$$
0=\lim _{\varepsilon \rightarrow 0} \lim _{n \rightarrow \infty}\left\langle J_{\lambda}^{\prime}\left(u_{n}, v_{n}\right),\left(u_{n} \phi_{\varepsilon}^{j}, v_{n} \phi_{\varepsilon}^{j}\right)\right\rangle \geq \tilde{\tau}_{x_{j}}-\tilde{v}_{x_{j}}
$$

By the Sobolev inequality, $S_{0} \tilde{v}_{x_{j}}^{\frac{2}{2^{*}}} \leq \tilde{\tau}_{x_{j}}$; and then we deduce that $\tilde{v}_{x_{j}}=0$ or $\tilde{v}_{x_{j}} \geq S_{0}^{\frac{N}{2}}$, which implies that $\mathcal{J}$ is finite.

Now, we consider the possibility of concentration at points $\xi_{i}(1 \leq i \leq k)$, for $\varepsilon>0$ small enough that $x_{j} \notin B_{\varepsilon}\left(\xi_{i}\right)$ for all $j \in \mathcal{J}$ and $B_{\varepsilon}\left(\xi_{i}\right) \cap B_{\varepsilon}\left(\xi_{j}\right)=\emptyset$ for $i \neq j$ and $1 \leq i, j \leq k$. Let $\varphi_{\varepsilon}^{i}$ be a smooth cut-off function centered at $\xi_{i}$ such that $0 \leq \varphi_{\varepsilon}^{i} \leq 1, \varphi_{\varepsilon}^{i}=1$ for $\left|x-\xi_{i}\right| \geq \varepsilon$ and $\left|\nabla \varphi_{\varepsilon}^{i}\right| \leq \frac{4}{\varepsilon}$. Then

$$
\begin{aligned}
& \begin{aligned}
& \lim _{\varepsilon \rightarrow 0} \lim _{n \rightarrow \infty} \int_{\Omega}\left(\left|\nabla u_{n}\right|^{2}+\left|\nabla v_{n}\right|^{2}\right) \varphi_{\varepsilon}^{i}=\lim _{\varepsilon \rightarrow 0} \int_{\Omega} \varphi_{\varepsilon}^{i} d \tilde{\tau} \\
& \geq \lim _{\varepsilon \rightarrow 0}\left(\int_{\Omega}\left(|\nabla u|^{2}+|\nabla v|^{2}\right) \varphi_{\varepsilon}^{i}+\tilde{\tau}_{\xi_{i}}\right) \\
&=\tilde{\tau}_{\xi_{i}}, \\
& \lim _{\varepsilon \rightarrow 0} \lim _{n \rightarrow \infty} \int_{\Omega} \frac{u_{n}^{2}+v_{n}^{2}}{\left|x-\xi_{i}\right|^{2}} \varphi_{\varepsilon}^{i}=\lim _{\varepsilon \rightarrow 0} \int_{\Omega} \varphi_{\varepsilon}^{i} d \tilde{\gamma}=\lim _{\varepsilon \rightarrow 0}\left(\int_{\Omega} \frac{u^{2}+v^{2}}{\left|x-\xi_{i}\right|^{2}} \varphi_{\varepsilon}^{i}+\tilde{\gamma}_{\xi_{i}}\right)=\tilde{\gamma}_{\xi_{i}}, \\
& \lim _{\varepsilon \rightarrow 0} \lim _{n \rightarrow \infty} \int_{\Omega}\left(\eta\left|u_{n}\right|^{2^{*}}+\lambda\left|v_{n}\right|^{2^{*}}+\sigma\left|u_{n}\right|^{\alpha}\left|v_{n}\right|^{\beta}\right) \varphi_{\varepsilon}^{i} \\
&=\lim _{\varepsilon \rightarrow 0} \int_{\Omega} \varphi_{\varepsilon}^{i} d \tilde{v}
\end{aligned}
\end{aligned}
$$




$$
\begin{aligned}
& \quad=\lim _{\varepsilon \rightarrow 0}\left(\int_{\Omega}\left(\eta|u|^{2^{*}}+\lambda|v|^{2^{*}}+\sigma|u|^{\alpha}|\nu|^{\beta}\right) \varphi_{\varepsilon}^{i}+\tilde{v}_{\xi_{i}}\right) \\
& \quad=\tilde{v}_{\xi_{i}}, \\
& \lim _{\varepsilon \rightarrow 0} \lim _{n \rightarrow \infty} \int_{\Omega} \frac{u_{n}^{2}+v_{n}^{2}}{\left|x-\xi_{i}\right|^{2}} \varphi_{\varepsilon}^{i}=0, \quad \text { for } i \neq j, \\
& \lim _{\varepsilon \rightarrow 0} \lim _{n \rightarrow \infty} \int_{\Omega}\left(u_{n} \nabla u_{n}+v_{n} \nabla v_{n}\right) \nabla \varphi_{\varepsilon}^{i}=0 .
\end{aligned}
$$

Thus, we have

$$
0=\lim _{\varepsilon \rightarrow 0} \lim _{n \rightarrow \infty}\left\langle J_{\lambda}^{\prime}\left(u_{n}, v_{n}\right),\left(u_{n} \varphi_{\varepsilon}^{i}, v_{n} \varphi_{\varepsilon}^{i}\right)\right\rangle \geq \tilde{\tau}_{\xi_{i}}-\mu_{i} \tilde{\gamma}_{\xi_{i}}-\tilde{\nu}_{\xi_{i}}
$$

From (4.1) and (4.2) we derive that $S_{\mu_{i}} \tilde{\nu}_{\xi_{i}}^{\frac{2}{2^{*}}} \leq \tilde{v}_{\xi_{i}}, 1 \leq i \leq k$, and then either $\tilde{v}_{\xi_{i}}=0$ or $\tilde{v}_{\xi_{i}} \geq$ $S_{\mu_{i}}^{\frac{N}{2}}$. On the other hand, from the above arguments, we conclude that

$$
\begin{aligned}
c & =\lim _{n \rightarrow \infty}\left(J\left(u_{n}, v_{n}\right)-\frac{1}{2}\left\langle J^{\prime}\left(u_{n}, v_{n}\right),\left(u_{n}, v_{n}\right)\right\rangle\right) \\
& =\frac{1}{N} \lim _{n \rightarrow \infty} \int_{\Omega}\left(\eta\left|u_{n}\right|^{2^{*}}+\lambda\left|v_{n}\right|^{2^{*}}+\sigma\left|u_{n}\right|^{\alpha}\left|v_{n}\right|^{\beta}\right) d x \\
& =\frac{1}{N}\left(\int_{\Omega}\left(\eta|u|^{2^{*}}+\lambda|v|^{2^{*}}+\sigma|u|^{\alpha}|v|^{\beta}\right) d x+\sum_{j \in \mathcal{J}} \tilde{v}_{x_{j}}+\sum_{i=1}^{k} \tilde{v}_{\xi_{i}}\right) \\
& =\frac{1}{N}\left(\sum_{j \in \mathcal{J}} \tilde{v}_{x_{j}}+\sum_{i=1}^{k} \tilde{v}_{\xi_{i}}\right)+J(u, v) .
\end{aligned}
$$

If $\tilde{v}_{\xi_{i}}=\tilde{v}_{x_{j}}=0$ for all $i \in\{1, \ldots, k\}$ and $j \in \mathcal{J}$, then $c=0$, which contradicts the assumption that $c>0$. On the other hand, if there exists an $i \in\{1, \ldots, k\}$ such that $\tilde{v}_{\xi_{i}} \neq 0$ or there exists a $j \in \mathcal{J}$ with $\tilde{v}_{x_{j}} \neq 0$, then we infer that

$$
c \geq \frac{1}{N} \min \left\{\left(S_{\eta, \lambda, \sigma}(0)\right)^{N / 2},\left(S_{\eta, \lambda, \sigma}\left(\mu_{1}\right)\right)^{N / 2}, \ldots,\left(S_{\eta, \lambda, \sigma}\left(\mu_{k}\right)\right)^{N / 2}\right\}=\frac{1}{N}\left(S_{\eta, \lambda, \sigma}\left(\mu_{k}\right)\right)^{N / 2},
$$

which contradicts our assumptions. Hence, $\left(u_{n}, v_{n}\right) \rightarrow(u, v)$, as $n \rightarrow \infty$ in $H \times H$.

First, under the assumptions $\left(\mathcal{H}_{1}\right),\left(\mathcal{H}_{2}\right)$, we have the following notations:

$$
\begin{aligned}
& f_{\eta, \lambda, \sigma}(\tau)=\frac{\left(1+\tau^{2}\right) S_{\mu_{k}}}{\left(\eta+\sigma \tau^{\beta}+\lambda \tau^{2^{*}}\right)^{\frac{2}{2^{*}}}}, \quad \tau>0 ; \\
& f_{\eta, \lambda, \sigma}\left(\tau_{\min }\right):=\min _{\tau>0} f_{\eta, \lambda, \sigma}(\tau)>0, \quad \sigma>0,
\end{aligned}
$$

where $\tau_{\min }>0$ is a minimal point of $f_{\eta, \lambda, \sigma}(\tau)$, and therefore a root of the equation

$$
\alpha \sigma \tau^{\beta}-\sigma \beta \tau^{\beta-2}-2^{*} \lambda \tau^{2^{*}-2}+2^{*} \eta=0, \quad \tau>0 .
$$

Lemma 4.2 Suppose that $\left(\mathcal{H}_{1}\right)$ holds. Then we have

(i) $S_{\eta, \lambda, \sigma}(\mu)=f_{\eta, \lambda, \sigma}\left(\tau_{\min }\right)$ 
(ii) $S_{\eta, \lambda, \sigma}(\mu)$ has the minimizers $\left(V_{\mu, \varepsilon}^{\xi}(x), \tau_{\min } V_{\mu, \varepsilon}^{\xi}(x)\right), \forall \varepsilon>0$, where $V_{\mu, \varepsilon}^{\xi}(x)$ are the extremal functions of $S_{\eta, \lambda, \sigma}(\mu)$ defined as in (2.2).

Proof The argument is similar to that of [6].

Lemma 4.3 Under the assumptions of $\left(\mathcal{H}_{1}\right)$, we have

$$
\sup J\left(t u_{\varepsilon, \mu_{k}}, t\left(\tau_{\min } u_{\varepsilon, \mu_{k}}\right)\right)<c^{*}=\frac{1}{N}\left(S_{\eta, \lambda, \sigma}\left(\mu_{k}\right)\right)^{N / 2} .
$$

Proof Suppose $\left(\mathcal{H}_{1}\right)$ holds. Define the function

$$
g(t):=J\left(t u_{\varepsilon, \mu_{k}}, t\left(\tau_{\min } u_{\varepsilon, \mu_{k}}\right)\right), \quad t \geq 0
$$

Note that $\lim _{t \rightarrow+\infty} g(t)=-\infty$ and $g(t)>0$ as $t$ is close to 0 . Thus, $\sup _{t \geq 0} g(t)$ is attained at some finite $t_{\varepsilon}>0$ with $g^{\prime}\left(t_{\varepsilon}\right)=0$. Furthermore, $c^{\prime}<t_{\varepsilon}<c^{\prime \prime}$, where $c^{\prime}$ and $c^{\prime \prime}$ are the positive constants independent of $\varepsilon$. By using (1.2), we have

$$
\begin{aligned}
g(t) \leq & \frac{t^{2}}{2}\left(1+\tau_{\min }^{2}\right)\left(\int_{\Omega}\left(\left|\nabla u_{\varepsilon, \mu_{k}}\right|^{2}-\mu_{k} \frac{u_{\varepsilon, \mu_{k}}^{2}}{\left|x-\xi_{i}\right|^{2}}-\lambda_{1} u_{\varepsilon, \mu_{k}}^{2}\right) d x\right) \\
& -\frac{t^{2^{*}}}{2^{*}}\left(\sigma \tau_{\min }^{\beta}+\eta+\lambda \tau_{\min }^{2^{*}}\right) \int_{\Omega}\left|u_{\varepsilon, \mu_{k}}\right|^{2^{*}} d x .
\end{aligned}
$$

Note that

$$
\max \left(\frac{t^{2}}{2} B_{1}-\frac{t^{2^{*}}}{2^{*}} B_{2}\right)=\frac{1}{N}\left(B_{1} B_{2}^{-2 / 2^{*}}\right)^{N / 2}, \quad B_{1}>0, B_{2}>0
$$

and $0 \leq \mu \leq \bar{\mu}-1$ and so $2<2 \sqrt{\bar{\mu}-\mu}$.

From (4.3), Lemma 4.2 and Lemma 4.3, it follows that

$$
\begin{aligned}
g\left(t_{\varepsilon}\right) & \leq \frac{1}{N}\left(\frac{\left(1+\tau_{\min }^{2}\right) \int_{\Omega}\left(\left|\nabla u_{\varepsilon, \mu_{k}}\right|^{2}-\mu_{k} \frac{u_{\varepsilon, \mu_{k}}^{2}}{\left|x-\xi_{k}\right|^{2}}-\lambda_{1} u_{\varepsilon, \mu_{k}}^{2}\right) d x}{\left.\left(\sigma \tau_{\min }^{\beta}+\eta+\lambda \tau_{\min }^{2^{*}}\right) \int_{\Omega}\left|u_{\varepsilon, \mu_{k}}\right|^{2^{*}} d x\right)^{2 / 2^{*}}}\right)^{N / 2} \\
& \leq \frac{1}{N}\left(\frac{f\left(\tau_{\min }\right)}{S\left(\mu_{k}\right)} \times \frac{\left(S\left(\mu_{k}\right)\right)^{N / 2}+O\left(\varepsilon^{2 \sqrt{\bar{\mu}-\mu}}\right)-C \varepsilon^{2}}{\left(S\left(\mu_{k}\right)\right)^{(N-2) / 2}+O\left(\varepsilon^{2 \sqrt{\mu}-\mu}\right)}\right) \\
& \leq \frac{1}{N}\left(f\left(\tau_{\min }\right)\right)^{N / 2}+O\left(\varepsilon^{2 \sqrt{\bar{\mu}-\mu}}\right)-C \varepsilon^{2} \\
& <\frac{1}{N}\left(S_{\eta, \lambda, \sigma}\left(\mu_{k}\right)\right)^{N / 2}=c^{*}
\end{aligned}
$$

so $g\left(t_{\varepsilon}\right)<c^{*}$. Hence, $g\left(t_{\varepsilon}\right)<c^{*}, \forall t \geq 0$ and

$$
\sup _{t \geq 0} g(t)=\sup _{t \geq 0} J\left(t u_{\varepsilon, \mu_{k}}, t\left(\tau_{\min } u_{\varepsilon, \mu_{k}}\right)\right)<c^{*}, \quad \text { if } \mu<\bar{\mu}-1 .
$$

Proof of Theorem 1.3 Set $c:=\inf _{h \in \Gamma} \max _{t \in[0,1]} J(h(t))$, where

$$
\Gamma=\{h \in C([0,1], H \times H) \mid h(0)=(0,0), J(h(1))<0\} .
$$


Suppose that $\left(\mathcal{H}_{1}\right)$ holds. For all $(u, v) \in H \times H \backslash\{(0,0)\}$, from the Young and HardySobolev inequalities, it follows that

$$
\begin{aligned}
J(u, v) & \geq C\left(\|u\|^{2}+\|v\|^{2}\right)-C\left(\|u\|^{2^{*}}+\|v\|^{2^{*}}\right) \\
& \geq C\|(u, v)\|^{2}-C\|(u, v)\|^{2^{*}},
\end{aligned}
$$

and there exists a constant $\rho>0$ small such that

$$
b:=\inf _{\|(u, v)\|=\rho} J(u, v)>0=J(0,0)
$$

Since $J(t u, t v) \rightarrow-\infty$ as $t \rightarrow \infty$, there exists $t_{0}>0$ such that $\left\|\left(t_{0} u, t_{0} v\right)\right\|>\rho$ and $J\left(t_{0} u, t_{0} v\right)<0$. By the mountain-pass theorem [14], there exists a sequence $\left\{\left(u_{n}, v_{n}\right)\right\} \subset$ $H \times H$ such that $J\left(u_{n}, v_{n}\right) \rightarrow c$ and $J^{\prime}\left(u_{n}, v_{n}\right) \rightarrow 0$, as $n \rightarrow \infty$.

From Lemma 4.2 it follows that

$$
\begin{aligned}
0<c & \leq \sup _{t \in[0,1]} J\left(t t_{0} u_{\varepsilon, \mu_{k}}, t t_{0} \tau_{\min } u_{\varepsilon, \mu_{k}}\right) \\
& \leq \sup _{t \geq 0} J\left(t u_{\varepsilon, \mu_{k}}, t \tau_{\min } u_{\varepsilon, \mu_{k}}\right) \\
& <c^{*} .
\end{aligned}
$$

By Lemma 4.1 there exists a subsequence of $\left\{\left(u_{n}, v_{n}\right)\right\}$, still denoted by $\left\{\left(u_{n}, v_{n}\right)\right\}$, such that $\left(u_{n}, v_{n}\right) \rightarrow(u, v)$ strongly in $H \times H$. Thus, we get a critical point $(u, v)$ of $J$ satisfying (1.1), and $c$ is a critical value. Set $u^{+}=\max \{u, 0\}$.

Replacing respectively $u, v$ with $u^{+}$and $v^{+}$in terms of the right-hand side of (1.1) and repeating the above process, we can get a nonnegative nontrivial solution $(u, v)$ of (1.1). If $u \equiv 0$, we get $v \equiv 0$ by (1.1) and the assumption $a_{2}>0$. Similarly, if $v \equiv 0$, we also have $u \equiv 0$. There, $u, v \neq \equiv$. From the maximum principle, it follows that $u, v>0$ in $\Omega$.

\section{Competing interests}

The authors declare that they have no competing interests.

\section{Authors' contributions}

Each of the authors, SK, MF and OKK contributed to each part of this work equally and read and approved the final version of the manuscript.

Received: 14 July 2012 Accepted: 4 October 2012 Published: 24 October 2012

\section{References}

1. Cao, D, Han, P: Solutions to critical elliptic equations with multi-singular inverse square potentials. J. Differ. Equ. 224, 332-372 (2006)

2. Hsu, TS: Multiple positive solutions for semilinear elliptic equations involving multi-singular inverse square potentials and concave-convex nonlinearities. Nonlinear Anal. 74, 3703-3715 (2011)

3. Kang, D: On the weighted elliptic problems involving multi-singular potentials and multi-critical exponents. Acta Math. Sin. Engl. Ser. 25, 435-444 (2009)

4. Abdellaoui, B, Felli, V, Peral, I: Some remarks on systems of elliptic equations doubly critical in the whole $\mathbb{R}^{N}$. Calc. Var. Partial Differ. Equ. 34, 97-137 (2009)

5. Bouchekif, M, Nasri, Y: On a singular elliptic system at resonance. Ann. Mat. Pura Appl. 189, 227-240 (2010)

6. Huang, Y, Kang, D: On the singular elliptic systems involving multiple critical Sobolev exponents. Nonlinear Anal. 74 400-412 (2011)

7. Kang, D: Semilinear systems involving multiple critical Hardy-Sobolev exponents and three singular points. Appl. Math. 218, 4514-4522 (2011)

8. Kang, D, Peng, S: Existence and asymptotic properties of solutions to elliptic systems involving multiple critical exponents. Sci. China Math. 54(2), 243-256 (2011) 
9. Kang, D, Huang, Y, Liu, S: Asymptotic estimates on the extremal functions of a quasi-linear elliptic problem. J. South-Central Univ. Natl. Nat. Sci. Ed. 27(3), 91-95 (2008)

10. Caffarelli, L, Kohn, R, Nirenberg, L: First order interpolation inequality with weights. Compos. Math. 53, 259-275 (1984)

11. Cai, M, Kang, D: Concentration-compactness principles for the systems of critical elliptic equations. Acta Math. Sci. Ser. B Engl. Ed. (to appear)

12. Lions, PL: The concentration-compactness principle in the calculus of variations: the limit case I. Rev. Mat. Iberoam. 1 , 45-121 (1985)

13. Lions, PL: The concentration-compactness principle in the calculus of variations: the limit case II. Rev. Mat. Iberoam. 1, 145-201 (1985)

14. Ambrosetti, A, Rabinowitz, PH: Dual variational methods in critical point theory and applications. J. Funct. Anal. 14, 349-381 (1973)

doi:10.1186/1687-2770-2012-119

Cite this article as: Khademloo et al.: Existence result for semilinear elliptic systems involving critical exponents.

Boundary Value Problems 2012 2012:119.

Submit your manuscript to a SpringerOpen ${ }^{\odot}$ journal and benefit from:

- Convenient online submission

- Rigorous peer review

- Immediate publication on acceptance

- Open access: articles freely available online

- High visibility within the field

- Retaining the copyright to your article

Submit your next manuscript at $\gg$ springeropen.com 